\title{
Lesões brancas benignas da mucosa oral: apresentação clínica, diagnóstico e
}

\section{tratamento}

Benign white lesions of the oral mucosa: clinical presentation, diagnosis and treatment

Lesiones blancas benignas de la mucosa oral: presentación clínica, diagnóstico y tratamento

Nívia Castro Binda

ORCID: https://orcid.org/0000-0002-0409-4265

Universidade Federal do Espírito Santo, Brasil E-mail: nivia_sgp@hotmail.com

Anne Caroline Silva Freire de Sá ORCID: https://orcid.org/0000-0003-3860-1373 Centro Universitário Tabosa de Almeida, Brasil E-mail: annecarolinefreiree@gmail.com

Thalita Oliveira da Silva Borba ORCID: https://orcid.org/0000-0001-5539-7744 Centro Universitário Tabosa de Almeida, Brasil E-mail: thalita.borba00@gmail.com Amanda Gonçalves Franco ORCID: https://orcid.org/0000-0003-0983-7539 Universidade de Itaúna, Brasil E-mail: amandagfranco38@gmail.com Jaqueline Lopes Reis

ORCID: https://orcid.org/0000-0003-1685-0336 Universidade de Rio Verde, Brasil E-mail: jaquelreis@hotmail.com Bruna Peixoto Girard

ORCID: https://orcid.org/0000-0003-0233-666X Centro Universitário Cesmac, Brasil E-mail: brunapeixoto5@hotmail.com

Nívia Delamoniky Lima Fernandes ORCID: https://orcid.org/0000-0001-8439-2117 Centro Universitário Inta, Brasil E-mail: delamonikynivia@gmail.com

Jefferson Douglas Lima Fernandes ORCID: https://orcid.org/0000-0002-5231-3813 Universidade Federal do Ceará, Brasil

E-mail: jefferson.odonto97@gmail.com José Victor Lima Silva

ORCID: https://orcid.org/0000-0002-8539-5456 Centro Universitário Christus, Brasil E-mail: victorlimasv@hotmail.com

Maria Vitória de Araúna Galvão ORCID: https://orcid.org/0000-0001-7312-3624 Centro Universitário Cesmac, Brasil E-mail: vitoria.arauna@gmail.com

Romulo de Oliveira Sales Junior ORCID: https://orcid.org/0000-0002-4638-7971

Centro Universitário UNINOVAFAPI, Brasil E-mail: romulojr_99@hotmail.com

Joana Machado Costa

ORCID: https://orcid.org/0000-0002-4421-2713 Universidade Tiradentes, Brasil E-mail: jmcbpinheiro@gmail.com

Thallita Monalisa Sizenando Souza Lima ORCID: https://orcid.org/0000-0003-1224-9420 Universidade Federal do Rio Grande do Norte, Brasil

E-mail: monalisa.sizenando.094@ufrn.edu.br

Thales Peres Candido Moreira ORCID: https://orcid.org/0000-0002-0622-3075 Universidade de São Paulo, Brasil E-mail: t.candido@usp.br 


\author{
Ana Luiza Castro Binda \\ ORCID: https://orcid.org/0000-0002-6664-2538 \\ Cirurgiã-Dentista, Brasil \\ E-mail: aninha_binda@hotmail.com
}

\begin{abstract}
Resumo
As lesões brancas benignas da cavidade oral apresentam alta recorrência e representam um grupo de entidades, cuja principal manifestação clínica se dá pela presença de áreas enbranquiçadas na boca. Apesar de haver características clássicas associadas a cada lesão, as aparências clinicas podem ser confundidas levando à um diagnóstico e tratamento incorreto. Diante do exposto, este trabalho possui como objetivo descrever as lesões brancas benignas mais comuns encontradas na mucosa oral, destacando sua etiologia, apresentação clínica, diagnóstico e tratamento. Para a construção deste trabalho foi feito um levantamento bibliográfico nas bases de dados SciVerse Scopus, Scientific Eletronic Library Online (Scielo), U.S. National Library of Medicine (PUBMED) e ScienceDirect, utilizando o gerenciador de referências Mendeley. A partir da revisão integrativa da literatura, observou-se que as lesões brancas benignas que afetam a mucosa oral com maior recorrência são a candidíase pseudomembranosa, leucoplasia, estomatite nicotínica, queilite actínica, leucoedema, líquen plano, reação de contato liquenóide, lesão por mordiscamento, linha alba e nevo branco esponjoso. Diante disso, é de extrema importância que os profissionais da suade estejam familiarizados com essas lesões, distinguindo-as de lesões malignas que afetam a cavidade oral, a afim de que se estabeleça o correto tratamento para cada caso.
\end{abstract}

Palavras-chave: Lesões brancas; Etiologia; Tratamento; Diagnóstico.

\begin{abstract}
Benign white lesions in the oral cavity have high recurrence and represent a group of entities whose main clinical manifestation is the presence of white areas in the mouth. Although there are classic features associated with each lesion, clinical appearances can be confounded, leading to incorrect diagnosis and treatment. Given the above, this work aims to describe the most common benign white lesions found in the oral mucosa, highlighting their etiology, clinical presentation, diagnosis and treatment. For the construction of this work, a bibliographic survey was carried out in the SciVerse Scopus, Scientific Electronic Library Online (Scielo), U.S. National Library of Medicine (PUBMED) and ScienceDirect databases, using the Mendeley reference manager. From the narrative review of the literature, it was observed that the most recurrent benign white lesions affecting the oral mucosa are pseudomembranous candidiasis, leukoplakia, nicotinic stomatitis, actinic cheilitis, leukoedema, lichen planus, lichenoid contact reaction, nibbling injury, line alba and white spongy nevus. Therefore, it is extremely important that health professionals are familiar with these lesions, distinguishing them from malignant lesions that affect the cavity, in order to establish the correct treatment for each case.
\end{abstract}

Keywords: White lesions; Etiology; Treatment; Diagnosis.

\title{
Resumen
}

Las lesiones blancas benignas en la cavidad bucal tienen alta recurrencia y representan un grupo de entidades cuya principal manifestación clínica es la presencia de áreas blancas en la boca. Aunque existen características clásicas asociadas con cada lesión, las apariencias clínicas pueden confundirse, lo que lleva a un diagnóstico y tratamiento incorrectos. Dado lo anterior, este trabajo tiene como objetivo describir las lesiones blancas benignas más comunes encontradas en la mucosa oral, destacando su etiología, presentación clínica, diagnóstico y tratamiento. Para la construcción de este trabajo se realizó un levantamiento bibliográfico en las bases de datos SciVerse Scopus, Scientific Electronic Library Online (Scielo), U.S. National Library of Medicine (PUBMED) y ScienceDirect, utilizando el gestor de referencias de Mendeley. A partir de la revisión narrativa de la literatura, se observó que las lesiones blancas benignas que afectan con mayor frecuencia a la mucosa oral son candidiasis pseudomembranosa, leucoplasia, estomatitis nicotínica, queilitis actínica, leucoedema, liquen plano, reacción de contacto liquenoide, lesión por mordisco, línea alba y nevo esponjoso blanco. Por tanto, es de suma importancia que los profesionales sanitarios conozcan estas lesiones, distinguiéndolas de las malignas que afectan a la cavidad, para poder establecer el tratamiento correcto para cada caso. Palabras clave: Lesiones blancas; Etiología; Tratamiento; Diagnóstico.

\section{Introdução}

As lesões da cavidade oral são subclassificadas em quatro grupos compreendendo ulcerações, pigmentações, lesões exofiticas e lesões branco-avermelhadas (McKinney et al., 2021)

As lesões brancas benignas da cavidade oral apresentam alta recorrência e representam um grupo de entidades, cuja principal manifestação clínica se dá pela presença de áreas esbranquiçadas na boca, geralmente em decorrência de uma produção exagerada de queratina. A respeito da etiologia, existem diversos fatores relacionados ao aparecimento de lesões brancas 
benignas na mucosa oral, como causas hereditárias, reativas, inflamatórias, imunológicas, traumáticas e infecções idiopáticas (Bhattacharyya \& Chehal, 2011; Mortazavi et al., 2019).

Em relação às características clínicas, as lesões brancas benignas podem assumir uma variedade de manifestações clinicas, desde simples alteração de desenvolvimento e assintomáticas, até grandes lesões com potencial de malignização, com elevado grau de displasia com complicações nas funções orais básicas do paciente, como fala, mastigação e deglutição. Apesar de haver características clássicas associadas a cada lesão, as manifestações clínicas podem ser confundidas levando à um diagnóstico e tratamento incorreto (Bhattacharyya \& Chehal, 2011; Mortazavi et al., 2019; Neville, 2009).

O profissional de saúde deve estar preparado para reconhecer o aspecto característico das lesões brancas que acometem a mucosa e saber diferenciá-las, sobretudo distinguir as lesões benignas de lesões malignas. Muitas das lesões benignas não exigem nenhum tratamento, somente a eliminação de fatores etiológicos. Em contrapartida, lesões brancas com potencial de se malignizar exigem maior atenção dos profissionais (Bhattacharyya \& Chehal, 2011).

Diante do exposto, este trabalho possui como objetivo descrever as lesões brancas benignas mais comuns encontradas na mucosa oral, destacando sua etiologia, apresentação clínica, diagnóstico e tratamento.

\section{Metodologia}

Refere-se a uma revisão integrativa de literatura, de caráter qualitativa. A revisão de literatura permite a busca aprofundada dentro de diversos autores e referenciais sobre um tema específico, nesse caso lesões brancas benignas da mucosa oral: apresentação clínica, diagnóstico e tratamento (Pereira et al., 2018).

A fim de que haja direcionamento na pesquisa, delineou-se como questão norteadora: "quais são as lesões brancas benignas que afetam a mucosa oral?", "quais são as suas manifestações clínicas" e "quais lesões devem ser excluídas no processo de diagnóstico?".

\subsection{Busca Estratégica}

Para a construção deste artigo foi feito um levantamento bibliográfico nas bases de dados SciVerse Scopus, Scientific Eletronic Library Online (Scielo), U.S. National Library of Medicine (PUBMED) e ScienceDirect, com auxílio do gerenciador de referência Mendeley. Os artigos foram coletados no período de maio a agosto de 2021 e contemplados entre os anos de 2015 a 2021.

A estratégia de pesquisa desenvolvida para identificar os artigos incluídos e avaliados para este estudo baseou-se nos descritores contidos na lista dos Descritores em Ciência da Saúde (DeCS) e suas combinações no idioma português e inglês: [(lesões brancas OR White lesions OR lesões benignas OR bening lesions) AND (mucosa oral OR oral mucosa OR boca OR mouth OR mucosa jugal OR cheek mucosa OR língua OR tongue) AND (manifestações clínicas OR clinical findings OR tratamento OR treatmente OR etiologia OR etiology OR diagnóstico OR diagnosis)]

\subsection{Critérios de inclusão e exclusão}

Considerou-se como critério de inclusão os artigos completos disponíveis na íntegra nas bases de dados citadas, nos idiomas inglês e português e relacionados com o objetivo deste estudo.

Os critérios de exclusão foram artigos incompletos, duplicados, resenhas, estudos in vitro e resumos.

\subsection{Seleção de estudos}

A estratégia de pesquisa baseou-se na leitura dos títulos para encontrar estudos que investigassem a temática da pesquisa. Caso atingisse esse primeiro objetivo, posteriormente, os resumos eram lidos e, persistindo na inclusão, era feita a leitura do 
artigo completo. Quando havia dúvida sobre a inclusão, o artigo era lido por outro autor e, a decisão de inclusão ou exclusão era tomada em consenso.

\subsection{Coleta de dados}

$\mathrm{Na}$ sequência metodológica foi realizada a busca e leitura na íntegra dos artigos pré-selecionados, os quais foram analisados para inclusão da amostra.

\section{Resultados e discussão}

Com base na revisão de literatura feita nas bases de dados eletrônicas citadas, foram identificados 1487 artigos científicos, dos quais 189 estavam duplicados com dois ou mais índices. Após a leitura e análise do título e resumos dos demais artigos outros 1203 foram excluídos. Assim, 81 artigos foram lidos na íntegra e, com base nos critérios de inclusão e exclusão, apenas 20 artigos foram selecionados para compor este estudo. O fluxograma com detalhamento de todas as etapas de seleção está na Figura 1.

Figura 1 - Fluxograma de identificação e seleção dos estudos.

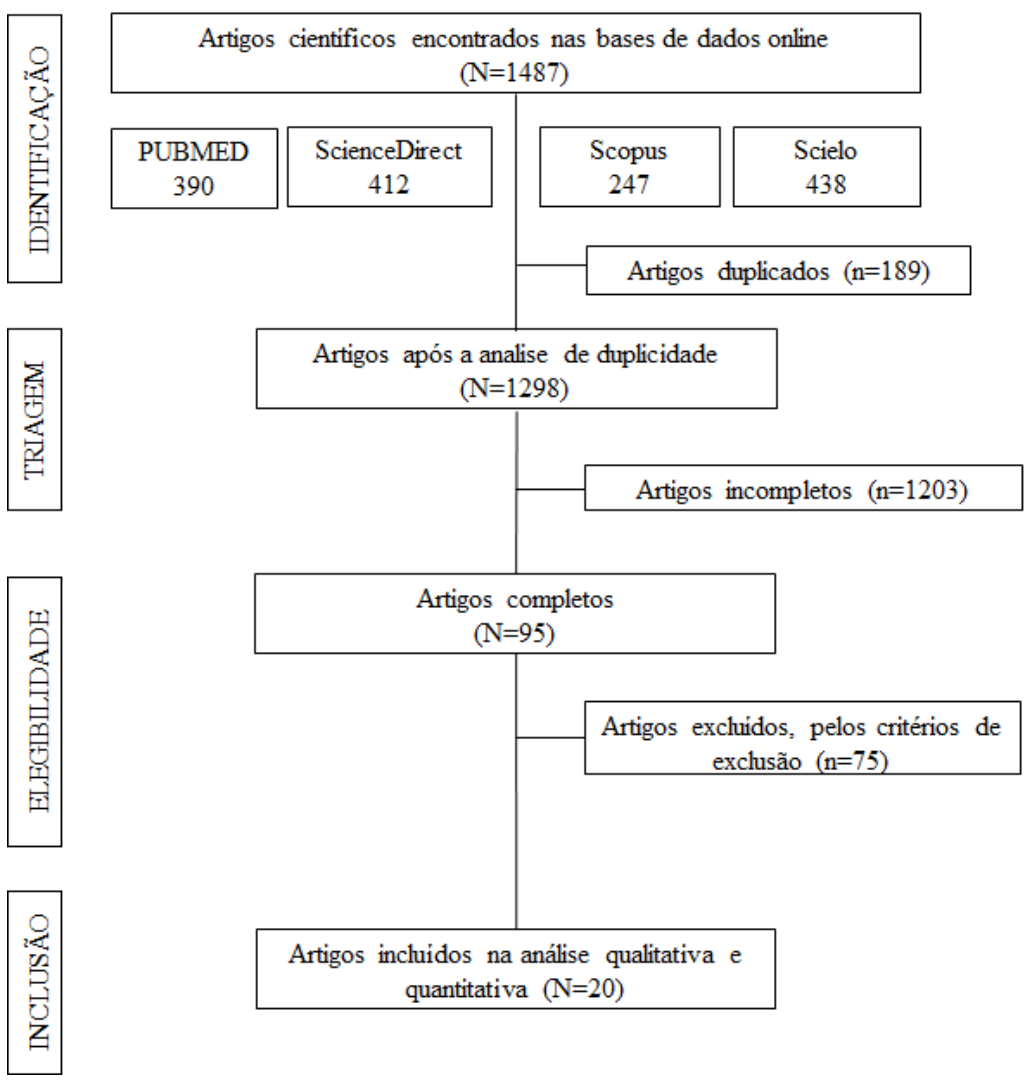

Fonte: Autoria própria (2021).

A partir da revisão integrativa da literatura, observou-se que as lesões brancas benignas que afetam a mucosa oral com maior recorrência são a candidíase pseudomembranosa, leucoplasia, estomatite nicotínica, queilite actínica, leucoedema, líquen plano, reação de contato liquenóide, lesão por mordiscamento, linha alba e nevo branco esponjoso. 


\subsection{Candidíase Pseudomembranosa}

A candidíase é uma infecção micótica mais frequente da cavidade oral, causada por fungos do gênero Cândida, mas comumente Cândida Albicans. Esse agente fúngico patogênico pode causar infecções superficiais na mucosa e infecções sistêmicas graves (Neville, 2009; Scully \& Porter, 2000).

Os fatores de risco associados a Candidíase envolvem idade mais avançada, infância ou gravidez, uso de antibiótico de largo espectro, uso de corticoesteróides inalatórios ou sistêmicos, má nutrição, com deficiente de vitaminas, malignidades, incluindo desordens hematológicas, xerostomia, causada irritação, síndrome de Sjögren e defeitos imunológicos, como HIV e síndrome da imunodeficiência adquirida (McKinney et al., 2021; Neville, 2009).

A candidíase pode manifestar-se de diversas formas na cavidade oral. Entretanto, a candidíase pseudomembranosa, também conhecida como sapinho, apresenta-se com maior recorrência. Clinicamente é caracterizada por placa branca aderida na região de mucosa labial e bucal, língua e palato, que se assemelham a queijo cottage ou leite colhado. As placas podem ser removidas pela raspagem pela fricção com uma compressa de gaze seca (McKinney et al., 2021; Neville, 2009).

A candidíase pseudomembranosa tem sido frequentemente associada ao uso de antibióticos de amplo espectro ou pela diminuição da capacidade imune do indivíduo. Quando há presença de sintomatologia, costuma ser relativamente brandos e descritos como uma sensação de queimação da mucosa bucal, acompanhado de um gosto desagradável (McKinney et al., 2021; Neville, 2009).

O diagnóstico é estabelecido por meio de achados clínicos; no entanto, como a candidíase pseudomembranosa pode acometer pacientes com doenças sistêmicas, um histórico médico deve ser avaliado para descartar qualquer causa subjacente. (McKinney et al., 2021; Neville, 2009).

O tratamento envolve o uso de medicamento antifúngico a ser prescrito pelo profissional da saúde, sendo o fluconazol ou clotrimazol como medicação de primeira escolha. Reforços aos hábitos de boa higiene oral, principalmente em pacientes que utilizam prótese dentária removível, deve ser realizados nesses pacientes para prevenir ou evitar a recorrência (McKinney et al., 2021; Neville, 2009).

\subsection{Leucoplasia}

É definida pela Organização Mundial da Saúde (OMS) como uma lesão hipoplásica não característica de qualquer outra doença. A Leucoplasia é a lesão oral pré-cancerosa mais frequente, representando cerca de $85 \%$ dos casos. O diagnóstico depende da exclusão de outras desordens que possuem as mesmas características clínicas (Neville, 2009; Ramos et al., 2017)

Geralmente atinge pessoas do sexo masculino (70\%) e acima de 40 anos. A causa da leucoplasia é desconhecida, porém existem várias hipóteses que podem contribuir para o surgimento da lesão, como o uso do tabaco, consumo excessivo de álcool, radiação ultravioleta e microrganismos, como o treponema pallidum, cândida albicans e papilomavirus humano (HPV) (Neville, 2009). A Tabela 1 descreve a classificação das leucoplasias quanto às suas características clínicas. 
Tabela 1 - Classificação das Leucoplasias

\begin{tabular}{clc}
\hline Classificação & \multicolumn{1}{c}{ Características Clínicas } & Locais mais frequentes \\
\hline \multirow{2}{*}{ Homogênea } & Lesão uniforme em cor e textura, predominantemente branca, Gengiva e mucosa jugal \\
& textura suave, fina ou levemente enrugada. Tipo mais comum \\
& e menos agressivo \\
\hline Não-homogênea & Superfície irregular ou verrucosa, podendo possuir áreas Ventre e borda de língua \\
& avermelhadas. \\
\hline
\end{tabular}

Fonte: Binda et al. (2021).

A leucoplasia oral é considerada uma lesão pré-maligna, com recorrência de malignização em 4 a $6 \%$ dos casos descritos na literatura. O exame histopatológico compõe uma ferramenta de extrema importância na confirmação do diagnóstico principal e na exclusão de outras lesões que apresentam características clinicas semelhantes (Mortazavi et al., 2019; Neville, 2009; Scully \& Porter, 2000).

A terapêutica é realizada por excisão cirúrgica, uso de betacaroteno, licopeno, ácido ascórbico, $\alpha$-tocoferol (vitamina E), ácido retinóico tópico e sistêmico (Vitamina A), bleomicina tópica e cirurgia a laser. Em virtude da leucoplasia ser uma desordem com potencial de transformação maligna para carcinoma epidermóide, a inspeção do paciente trimestral ou semestral é obrigatória por toda a vida (Neville, 2009; Scully \& Porter, 2000).

\subsection{Estomatite Nicotínica}

Também chamada de nicotina palatina ou palato do tabagista, a estomatite nicotínica (EN) consiste em uma lesão branca comum devido a hábito de fumar. Tal desordem é encontrada com maior frequência em homens, com idade superior a 45 anos que fazem o uso de cachimbo, tabaco, cigarro e em fumantes reversos (Bhattacharyya \& Chehal, 2011; Mortazavi et al., 2019; Neville, 2009).

Clinicamente, a mucosa palatina apresenta-se com área branca acinzentada difusa, podendo estar acompanhada de múltiplas pápulas que representam os orifícios alargados e inchados das glândulas salivares menores presentes nessa região. Em alguns casos, a placa branca pode afetar as gengivas marginais e as papilas interdentais (Bhattacharyya \& Chehal, 2011; Mortazavi et al., 2019; Neville, 2009).

A EN é completamente reversível, dispensando a necessidade de tratamento. A lesão tende a regredir totalmente dentro de uma a duas semanas após o fim do hábito de fumar. Apesar da estomatite nicotínica não ser considerada como uma desordem pré-maligna o palato do fumante reverso tem potencial considerável para transformação maligna. Sendo assim, qualquer lesão branca da mucosa palatina durando mais de um mês após a remoção de hábitos nocivos deve ser cuidadosamente monitorada para descartar a malignidade (Bhattacharyya \& Chehal, 2011; Mortazavi et al., 2019; Neville, 2009).

\subsection{Queilite Actínica}

A queilite actínica ou queilose actínica é uma desordem pré-maligna comum do vermelho do lábio inferior resultante de uma exposição exacerbada ao espectro ultravioleta da luz solar; no entanto outros fatores de risco possuem associação, como idade avançada, pele clara imunossupressão exposição ao arsênio e anormalidades genéticas (Mortazavi et al., 2019; Neville, 2009).

Em relação à apresentação clínica, a queilite actínica manifesta-se, primariamente, como secura excessiva, inchaço e rachaduras com regiões atróficas claras, manchadas e lisas no vermelho do lábio inferior, com perda dos limites entre o vermelhão 
e porção cutânea do lábio subjacente. Com evolução da lesão, nota-se áreas ásperas, escamosas, com crostas e placas ceratóticas. Por fim, a ulceração central aparece em um ou mais sítios e podem permanecer por meses e frequentemente progride para lesões malignas (Mortazavi et al., 2019; Neville, 2009; Vieira et al., 2012).

Com relação ao diagnóstico da queilite actínica, deve ser realizado a biopsia nas áreas afetadas para exclusão da possibilidade de carcinoma. Nos casos em que a lesão for grave, porém sem transformação maligna, torna-se necessário o procedimento cirúrgico com a técnica de shave labial (vermelhonectomia). Tratamentos alternativos envolvem o uso de laser de $\mathrm{CO} 2$, eletrodissecção, creme de 5-fluorouracil, aplicação tópica de imiquimod, terapia fotodinâmica e quimioexfoliação com ácido tricloroacético. O acompanhamento de longa duração é recomendado em casos de queilite actínica (Neville, 2009)

\subsection{Leucoedema}

Consiste em um achado clínico comum na cavidade oral, de etiologia desconhecida. Acomete, predominantemente, pessoas negras, sem predileção por sexo, sustentando a probabilidade de predisposição genética para o seu desenvolvimento. Por ser muito comum, o leucodema é melhor classificado como uma variação da normalidade do que uma doença, dispensando qualquer tipo de tratamento (Mortazavi et al., 2019; Neville, 2009).

Clinicamente, apresenta-se, bilateralmente, com aparência difusa, opalescente e branco-acinzentada cremosa da mucosa. A superfície apresenta-se frequentemente pregueada, resultando em estrias esbranquiçadas ou rugosidades, não destacáveis a raspagem e sem sintomatologia. Um critério clinico importante para o diagnóstico do leucoedema é o fato de ao distender e everter a mucosa oral, o aspecto esbranquiçado desaparece temporariamente (Mortazavi et al., 2019; Neville, 2009).

\subsection{Líquen Plano}

O líquen plano (LP) é uma doença dermatológica crônica que, com alta recorrência, afeta a mucosa oral. Sua etiologia permanece desconhecida, porém alguns fatores têm sido associados, como ansiedade, diabetes, doenças autoimunes, doenças intestinais, uso de drogas e estresse (Bakhtiari et al., 2017; Mortazavi et al., 2019; Neville, 2009).

Clinicamente, a lesão é descrita como pápulas poligonais púrpuras e pruriginosas. Três formas de lesões bucais de líquen plano oral (LPO) são descritas: líquen plano reticular, líquen plano erosivo e líquen plano bolhoso (Neville, 2009).

O líquen plano reticular não possui sintomatologia dolorosa e manifesta-se na região posterior da mucosa jugal, bilateralmente, borda lateral e dorso da língua, a gengiva, o palato, e o vermelhão labial. Apresenta-se como estrias brancas entrelaçadas, chamadas de estrias de Wickam (Bakhtiari et al., 2017; Mortazavi et al., 2019; Neville, 2009).

Já o líquen plano erosivo, apesar de não ser tão frequente quanto o reticular, necessita de um maior cuidado, devido a apresentação de sintomatologia dolorosa. Ao exame físico intrabucal é possível notar áreas eritematosas atróficas, com graus variáveis de ulceração central. Se o componente erosivo for grave, pode ocorrer separação entre o epitélio e o tecido conjuntivo subjacente, resultando na apresentação relativamente rara do líquen plano bolhoso (Mortazavi et al., 2019; Neville, 2009; Scully \& Porter, 2000). A Tabela 2 descreve a classificação do LPB de acordo com suas características clínicas e sintomatologia. 
Tabela 2 - Classificação do Líquen Plano Bucal.

\begin{tabular}{|c|c|c|c|}
\hline Tipo & Considerações clínicas & Sítio de acometimento & Sintomatologia \\
\hline $\begin{array}{c}\text { Forma } \\
\text { Reticular }\end{array}$ & $\begin{array}{l}\text { Linhas brancas entrelaçadas } \\
\text { (estrias de Wickham), envoltas por } \\
\text { uma borda eritematosa. } \\
\text { Forma mais recorrente. }\end{array}$ & $\begin{array}{l}\text { Região posterior da mucosa jugal } \\
\text { bilateralmente. Podendo acometer, } \\
\text { concomitantemente, a borda lateral e o } \\
\text { dorso da língua, gengiva, palato, e o } \\
\text { vermelho do lábio }\end{array}$ & Assintomático \\
\hline $\begin{array}{l}\text { Forma } \\
\text { Erosiva }\end{array}$ & $\begin{array}{l}\text { Áreas atróficas e eritematosas } \\
\text { envoltas por estrias irradiadas, com } \\
\text { graus variáveis de ulceração central }\end{array}$ & Qualquer região da mucosa oral. & Dor ou queimação no local \\
\hline $\begin{array}{l}\text { Forma } \\
\text { Bolhosa }\end{array}$ & $\begin{array}{l}\text { Se a lesão for muito grave, poderá } \\
\text { haver a separação do epitélio e o } \\
\text { tecido conjuntivo, gerando a forma } \\
\text { bolhosa }\end{array}$ & $\begin{array}{l}\text { Mucosa jugal nas regiões posterior e } \\
\text { inferior dos } 2^{\circ} \text { e } 3^{\circ} \text { molares. }\end{array}$ & $\begin{array}{l}\text { Dor no local, principalmente } \\
\text { quando há rompimento das } \\
\text { bolhas }\end{array}$ \\
\hline
\end{tabular}

Fonte: Binda et al. (2021).

Com relação ao tratamento do líquen plano, a literatura aponta que a forma reticular não necessita, na maioria das vezes, de tratamento. Em alguns casos a sobreposição de espécies da Cândida pode causar sensação de queimação na cavidade oral, o que torna necessário o uso de antifúngicos (Mortazavi et al., 2019; Neville, 2009; Scully \& Porter, 2000).

Em contrapartida, a forma erosiva e bolhosa, devem seguir a terapia medicamentosa com corticoesteróides tópicos concomitante de drogas antifúngicas, devido a sintomatologia dolorosa relatada pelos pacientes (Pereda Rojas et al., 2016). Os esteroides sistêmicos são recomendados para reduzir os sintomas de lesões resistentes. Outros tratamentos envolvem inibidores de calcineurina, retinóides e fototerapia ultravioleta (Bakhtiari et al., 2017; Mortazavi et al., 2014).

O LPO é considerado uma desordem potencialmente maligna de baixo risco, representando cerca de $0,2 \%$ ao ano de transformação maligna. Dessa forma, o monitoramento anual deve ser feito em pacientes com LPO. A literatura aponta que placas, lesões erosivas e ulcerativas, principalmente na região de palato mole, borda lateral e ventre de língua e assoalho de boca são sítios com maior tendência para transformação maligna, e a biopsia deve ser considerada para excluir displasia ou carcinoma (Neville, 2009; Nosratzehi, 2018).

\subsection{Reação de contato Liquenóide}

As reações de contato liquenóide (RCL) são caracterizadas como uma reação de hipersensibilidade decorrente de constituintes derivados de materiais dentários. Habitualmente, possui caráter assintomático, porém pode haver queixas de desconforto durante a mastigação de alimentos quentes em casos em que a lesão apresenta sua eritematosas ou ulcerativas (Kamath et al., 2015; Neville, 2009).

Clinicamente, as RCLs apresentam as mesmas características do líquen plano oral, como pápulas, retículos, eritema e úlceras. Entretanto, a extensão e local da lesão permite diferenciar tais desordens. A maioria das RCLs está restrita a regiões de contato com materiais dentários, como a mucosa bucal e bordas laterais da língua, sendo raramente encontradas em gengivas, mucosa palatina, assoalho da boca e ventre de língua (Neville, 2009).

O diagnóstico das RCLs é estabelecido através do exame físico da mucosa oral e sua relação direta com restaurações dentárias. Em alguns casos, a biopsia pode ser realizada para confirmar o diagnóstico e para eliminar outras patologias, como a 
displasia. Entretanto, as características histopatológicas encontradas no RCLs são indistinguíveis das do líquen plano oral (Neville, 2009)

O tratamento das RCLs consiste em medidas locais, como melhora na higiene oral, alisamento, polimento e recontorno das restaurações. Em alguns casos, deve realizar a remoção do fator de irritação e substitui-lo por restauração não metálica (Neville, 2009)

\subsection{Lesão por Mordiscamento}

A lesão por mordiscamento é comum em pessoas ansiosas e que passam por situações de estresse. A lesão é encontrada, normalmente, na mucosa jugal, bilateralmente, ou nas margens laterais da língua. Acomete, em sua maioria, mulheres acima de 35 anos de idade (Anura, 2014; Kang et al., 2012).

Clinicamente, é possível notar uma área branca com espessamento e descamação do epitélio, podendo haver áreas eritematosas, com erosão ou ulceração traumática. De forma clássica, essas lesões acometem a mucosa jugal, em sua porção média, na região correspondente a linha de oclusão dentária (Anura, 2014; Kang et al., 2012; Mortazavi et al., 2019).

Por se tratar de uma lesão benigna que não apresenta riscos de complicações, a lesão por mordiscamento não requer nenhum tratamento. A regressão se baseia na eliminação de hábitos nocivos, como morder a mucosa bucal (Anura, 2014; Kang et al., 2012; Mortazavi et al., 2019; Neville, 2009).

\subsection{Linha alba}

Também chamada de linha de mordida horizontal, a linha alba é uma alteração benigna muito comum da mucosa bucal. A fisiopatologia da lesão envolve uma combinação de irritação por atrito e trauma por sucção ao longo das superfícies vestibulares e ao longo das superfícies olcusais opostas (Messadi et al., 2003; Neville, 2009).

Ao exame físico intrabucal, observa-se uma linha branca, bilateral, na mucosa jugal ao longo do plano oclusal dos dentes adjacentes. A linha varia em proeminência de pouco visível a altamente proeminente (Messadi et al., 2003; Neville, 2009).

O diagnóstico da linha alba é realizado pelo exame físico, dispensando a biopsia no local. Por se tratar de uma lesão benigna sem complicações, intervenções terapêuticas não são necessárias. Entretanto, em alguns casos, pode ser recomendado a utilização de telas de mordida, para serem utilizadas durante a noite, a fim de proteger a mucosa jugal de mordidas involuntárias (McKinney et al., 2021; Messadi et al., 2003; Neville, 2009).

\subsection{Nevo branco esponjoso}

O nevo branco esponjoso (NBE), também chamado de doença de Cannon e displasia pregueada branca familial, consiste em uma doença autossômica dominante relativamente rara, sem predileção por sexo, resultante de um defeito na ceratinização da mucosa bucal (Neville 2009).

Clinicamente o NBE manifesta-se como placa branca simétricas, espessas, difusas, corrugadas ou aveludadas, de tamanhos variados, sem presença de sintomatologia dolorosa. A mucosa jugal, bilateralmente, é a área mais acometida pela lesão, entretanto, áreas como ventre de língua, mucosa labial, palato mole, mucosa alveolar e assoalho de boca também podem estarem acometidos. Lesões extraorais, como mucosa genital, laríngea e esofágica são incomuns (de Haseth et al., 2017; Neville, 2009).

Por se tratar de uma lesão hereditária, o diagnóstico do NBE é realizado com base na história detalhada do paciente e da família, além de inspeção das lesões nas mucosas afetadas, analise histopatológica e análise genética molecular. Por se tratar de uma lesão benigna, nenhum tratamento é indicado para NBE (Ali et al., 2013; de Haseth et al., 2017; Neville, 2009) . 


\subsection{Características que ajudam no diagnóstico}

A Tabela 3 descreve de forma simplificada o sítio anatômico mais frequente para cada uma das lesões citadas e seus principais meios de diagnósticos.

Tabela 3 - Características que ajudam no diagnóstico de lesões brancas benignas da mucosa oral.

\begin{tabular}{|c|c|c|}
\hline Lesão & Sítio Anatômico & Principais meios de diagnóstico \\
\hline $\begin{array}{c}\text { Candidíase } \\
\text { Pseudomembranosa }\end{array}$ & Boca, língua e palato & 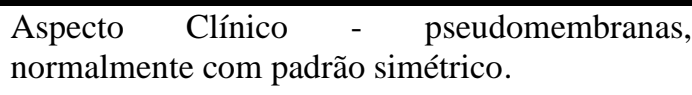 \\
\hline Leucoplasia & $\begin{array}{l}\begin{array}{l}\text { mucosa bucal, vermelhão labial e } \\
\text { gengiva }\end{array} \\
\end{array}$ & Aspecto clínico e eliminação de outras desordens. \\
\hline $\begin{array}{l}\text { Estomatite } \\
\text { Nicotínica }\end{array}$ & Mucosa palatina & Aspecto clínica e histórico de hábitos de fumar. \\
\hline $\begin{array}{l}\text { Queilite } \\
\text { Actínica }\end{array}$ & Vermelhão do lábio inferior & Aspecto clínico e biopsia. \\
\hline Leucoedema & Bucal & Aspecto clínico e extensão da mucosa. \\
\hline Líquen Plano & Mucosa bucal posterior bilateralmente & $\begin{array}{l}\text { Aspecto clínico (normalmente possui padrão } \\
\text { simétrico) e histopatologia. }\end{array}$ \\
\hline $\begin{array}{l}\text { Reação de contato } \\
\text { liquenóide }\end{array}$ & $\begin{array}{l}\text { Locais que estão regularmente em } \\
\text { contato com materiais dentários (mucosa } \\
\text { bucal e bordas laterais da língua) }\end{array}$ & $\begin{array}{l}\text { Aspecto clínico (relação com restauração } \\
\text { metálica) e, em alguns casos, biopsia. }\end{array}$ \\
\hline Lesão por mordiscamento & $\begin{array}{l}\text { Mucosa jugal, lábios, borda lateral da } \\
\text { língua }\end{array}$ & $\begin{array}{l}\text { Aspecto clínico e anamnese (história de hábitos } \\
\text { de mordida ou mastigação). }\end{array}$ \\
\hline Linha Alba & Mucosa jugal & Aspecto clínico \\
\hline $\begin{array}{l}\text { Nevo Branco } \\
\text { Esponjoso }\end{array}$ & mucosa jugal, & $\begin{array}{l}\text { Histórico do paciente e da sua família, exames } \\
\text { clinico e histopatológico e análise genética } \\
\text { molecular. }\end{array}$ \\
\hline
\end{tabular}

Fonte: autoria própria, (2021).

\subsection{Diagnósticos Diferenciais}

A Tabela 4 aborda as lesões brancas benignas da mucosa oral, simplificando seus diagnósticos diferenciais e tratamentos indicados. 
Tabela 4 - Diagnósticos diferencial e tratamento das lesões brancas benignas da cavidade oral.

\begin{tabular}{|c|c|c|}
\hline Lesão & Diagnóstico Diferencial & Tratamento \\
\hline $\begin{array}{c}\text { Candidíase } \\
\text { Pseudomembranosa }\end{array}$ & Líquen Plano, trauma e deficiência nutricional. & Antifúngicos. \\
\hline Leucoplasia & $\begin{array}{l}\text { Líquen plano, candidíase pseudomembranosa e } \\
\text { lúpus eritematoso crônico discóide }\end{array}$ & Não indicado \\
\hline Estomatite Nicotínica & Leucoplasia, candidíase e penfigóide bolhoso. & Regressão após cessação do tabagismo. \\
\hline Nevo Branco esponjoso & $\begin{array}{l}\text { Lúpus eritematoso crônico discóide e outros } \\
\text { tipos de queilites (esfoliativa, glangular e } \\
\text { granulomatosa) }\end{array}$ & Não indicado \\
\hline Leucoedema & $\begin{array}{l}\text { Leucoplasia, nevo branco esponjoso, lesão por } \\
\text { mordiscamento e hiperqueratose }\end{array}$ & Não indicado \\
\hline Líquen Plano Oral & Pênfigo, leucoedema e leucoplasia. & $\begin{array}{l}\text { Esteróide tópico, uso concomitante de } \\
\text { drogas antifúngicas. }\end{array}$ \\
\hline $\begin{array}{l}\text { Reação de contato } \\
\text { liquenóide }\end{array}$ & Líquen plano oral, leucoplasia e candidíase & $\begin{array}{l}\text { Melhora na higiene oral, alisamento, } \\
\text { polimento e recontorno das restaurações. } \\
\text { Substituição do material restaurador. }\end{array}$ \\
\hline $\begin{array}{l}\text { Lesão por } \\
\text { mordiscamento }\end{array}$ & $\begin{array}{l}\text { Líquen plano oral, candidíase, leucoplasia, nevo } \\
\text { branco esponjoso }\end{array}$ & Cessação da mastigação habitual. \\
\hline Linha Alba & $\begin{array}{l}\text { Lesão por mordiscamento, leucoedema e líquen } \\
\text { plano oral. }\end{array}$ & $\begin{array}{l}\text { Cessação do hábito nocivo e utilização de } \\
\text { placas dentárias noturnas. }\end{array}$ \\
\hline Nevo Branco Esponjoso & $\begin{array}{l}\text { Leucoedema, líquen plano oral e lesão por } \\
\text { mordiscamento. }\end{array}$ & Não indicado \\
\hline
\end{tabular}

Fonte: autoria própria, (2021).

\section{Considerações finais}

A partir das trajetórias bibliográficas dos pesquisadores, esse estudo abordou acerca das lesões brancas benignas da mucosa oral, destacando sua apresentação clínica, seus diagnósticos e seus tratamentos.

Os resultados desta pesquisa revelam dez principais lesões brancas na mucosa oral, sendo elas candidíase pseudomembranosa, leucoplasia, estomatite nicotínica, queilite actínica, leucoedema, líquen plano, reação de contato liquenóide, lesão por mordiscamento, linha alba e nevo branco esponjoso. Diante disso, é de extrema importância que os profissionais da suade estejam familiarizados com essas lesões, distinguindo-as de lesões malignas que afetam a cavidade ora, a afim de que se estabeleça o correto tratamento para cada caso.

Com isso, o levantamento bibliográfico sobre as lesões brancas benignas da mucosa oral se mostra importante, pois envolve estudos retrospectivos que possuem relação com o tema proposto e, ao mesmo, tempo, cria-se a possibilidade futura de novos pesquisadores abordarem o tema, criando novos trabalhos, para que haja melhor direcionamento quanto ao diagnóstico e tratamento correto dessas lesões.

\section{Referências}

Ali, M., Joseph, B., \& Sundaram, D. (2013). Prevalence of oral mucosal lesions in patients of the Kuwait University Dental Center. The Saudi Dental Journal, 25(3), 111-118. https://doi.org/10.1016/j.sdentj.2013.05.003

Anura, A. (2014). Traumatic oral mucosal lesions: a mini review and clinical update. Oral Health and Dental Management, 13(2), $254-259$.

Bakhtiari, S., Azari-Marhabi, S., Mojahedi, S. M., Namdari, M., Rankohi, Z. E., \& Jafari, S. (2017). Comparing clinical effects of photodynamic therapy as a 
novel method with topical corticosteroid for treatment of Oral Lichen Planus. Photodiagnosis and Photodynamic Therapy, 20, 159-164. https://doi.org/10.1016/j.pdpdt.2017.06.002

Bhattacharyya, I., \& Chehal, H. K. (2011). White lesions. Otolaryngologic Clinics of North America, 44(1), 109-131, vi. https://doi.org/10.1016/j.otc.2010.09.009

Binda, N. C., Binda, A. L. C., Pinho, R. A. de, Ramalho, M. A., Leão, G. C., Girard, B. P., Silva, R. B., Silva, M. K. G. da, Fernandes, N. D. L., Fernandes, J. D. L., Sousa, Z. da S., Monteiro, M. S., Coêlho, L. P. I., Barros, L. S. de A., Moreira, T. P. C., Costa, A. M., \& Leão, M. J. da R. (2021). Lesões potencialmente malignas da região bucomaxilofacial. In Research, Society and Development (Vol. 10, Issue 11, p. e185101119452). https://doi.org/10.33448/rsd-v10i11.19452

de Haseth, S. B., Bakker, E., Vermeer, M. H., El Idrissi, H., Bosse, T., Smit, V. T. H. B. M., Terron-Kwiatkowski, A., McLean, W. H. I., Peters, A. A. W., \& Hes, F. J. (2017). A novel keratin 13 variant in a four-generation family with white sponge nevus. Clinical Case Reports, 5(9), 1503-1509. https://doi.org/10.1002/ccr3.1073

Kamath, V. V., Setlur, K., \& Yerlagudda, K. (2015). Oral lichenoid lesions - a review and update. Indian Journal of Dermatology, 60(1), 102. https://doi.org/10.4103/0019-5154.147830

Kang, H. S., Lee, H. E., Ro, Y. S., \& Lee, C. W. (2012). Three cases of “morsicatio labiorum”. In Annals of dermatology (Vol. 24, Issue 4, pp. 455-458). https://doi.org/10.5021/ad.2012.24.4.455

McKinney, R., Olmo, H., \& McGovern, B. (2021). Benign Chronic White Lesions Of The Oral Mucosa.

Messadi, D. V., Waibel, J. S., \& Mirowski, G. W. (2003). White lesions of the oral cavity. Dermatologic Clinics, 21(1), 63-78. https://doi.org/https://doi.org/10.1016/S0733-8635(02)00069-4

Mortazavi, H., Baharvand, M., \& Mehdipour, M. (2014). Oral potentially malignant disorders: an overview of more than 20 entities. Journal of Dental Research, Dental Clinics, Dental Prospects, 8(1), 6-14. https://doi.org/10.5681/joddd.2014.002

Mortazavi, H., Safi, Y., Baharvand, M., Jafari, S., Anbari, F., \& Rahmani, S. (2019). Oral White Lesions: An Updated Clinical Diagnostic Decision Tree. Dentistry Journal, 7(1), 15. https://doi.org/10.3390/dj7010015

Neville, B. W. D. D. C. A. J. B. (2009). Oral and Maxillofacial Pathology. (3rd ed.).

Nosratzehi, T. (2018). Oral Lichen Planus: an Overview of Potential Risk Factors, Biomarkers and Treatments. Asian Pacific Journal of Cancer Prevention : APJCP, 19(5), 1161-1167. https://doi.org/10.22034/APJCP.2018.19.5.1161

Pereda Rojas, M. E., González Cardona, Y., \& Torres Herrera, L. W. (2016). Actualización sobre liquen plano bucal. Correo Científico Médico, 20(3), 539_ 555 .

Pereira, A., Shitsuka, D., Parreira, F., \& Shitsuka, R. (2018). Método Qualitativo, Quantitativo ou Quali-Quanti. In Metodologia da Pesquisa Científica. https://repositorio.ufsm.br/bitstream/handle/1/15824/Lic_Computacao_Metodologia-Pesquisa-Cientifica.pdf?sequence=1. Acesso em: 28 março 2020.

Ramos, R. T., Paiva, C. R., Filgueiras, A. M. D. O., Silva-Junior, G. O., Cantisano, M. H., Ferreira, D. D. C., \& Ribeiro, M. (2017). Leucoplasia Oral: conceitos e repercussões clínicas. Revista Brasileira de Odontologia, 74(1), 51. https://doi.org/10.18363/rbo.v74n1.p.51

Scully, C., \& Porter, S. (2000). ABC of oral health. Swellings and red, white, and pigmented lesions. BMJ (Clinical Research Ed.), 321(7255), 225-228. https://doi.org/10.1136/bmj.321.7255.225

Vieira, R. A. M. A. R., Minicucci, E. M., Marques, M. E. A., \& Marques, S. A. (2012). Actinic cheilitis and squamous cell carcinoma of the lip: clinical, histopathological and immunogenetic aspects. Anais Brasileiros de Dermatologia, 87(1), 105-114. https://doi.org/10.1590/s0365-05962012000100013 